\title{
Utilization of Both Odd and Even Modes for Multi-band Dipole Antenna
}

\author{
Bing Xiao, Hang Wong, Min Li, Bo Wang, and Kwan L. Yeung
}

\begin{abstract}
Dipole antenna is the simplest type of antenna from the theoretical point of view. However, the majority of the research on it focuses on adding supplementary structures for producing more resonant modes to achieve more functions. Here, we focus on the inherent modes of the dipole itself. The dipole antenna has alternating odd and even modes. In classical antenna theory, only odd modes are excited. The even modes are difficult to be excited due to their very high input impedances. In this paper, both odd and even modes of the dipole antenna are excited simultaneously by a single port with a novel feeding structure. Moreover, in order to tune the resonant frequencies of such multiple modes, a method by combining antithetical structures of indentations and outdentations is proposed and intuitively explained by eigencurrent distribution. By these new techniques, all the first six modes of the dipole antenna are excited, among which five modes are utilized for IoT applications. The antenna could cover ISM bands of $868 \mathrm{MHz}$ and $2450 \mathrm{MHz}$, GNSS band of $1575 \mathrm{MHz}$, and $5 \mathrm{G}$ bands of $3500 \mathrm{MHz}$ and $4900 \mathrm{MHz}$. This research could broaden the application of this classic antenna.
\end{abstract}

Index Terms-dipole antenna, even mode, Internet of Things (IoT), multi-band, multi-mode, odd mode, theory of characteristic mode (TCM)

\section{INTRODUCTION}

$\mathrm{A}^{\mathrm{t}}$ present, many of the objects surrounding us are being connected to the Internet of Things (IoT). Various IoT communication protocols coexist, operating on different frequencies, such as cellular networks from $2 \mathrm{G}$ to $5 \mathrm{G}$ (especially the $5 \mathrm{G}$ massive machine-type communication), Low-Power Wide-Area Network (LPWAN), Bluetooth and its derived Bluetooth Low Energy (BLE), Wireless Local Area Network (WLAN), Radio-Frequency Identification (RFID), etc. On the other hand, an IoT gateway is a network node that connects the above-mentioned multiple networks with different protocols [1]. Some wireless smart devices, such as

This work was supported in part by the Research Grants Council of the Hong Kong SAR, China with a TRS project (Project No. T42-103/16-N), a CRF project (Project No. CityU C1020-19E), and the CityU Strategic Research Grant (Project No. SRG-Fd 7005227).

Bing Xiao and L. K. Yeung are with the Department of Electrical and Electronic Engineering, The University of Hong Kong, Hong Kong.

H. Wong is with The State Key Laboratory of Terahertz and Millimeter Waves, and Shenzhen Research Institute, Department of Electrical Engineering, City University of Hong Kong, Hong Kong (e-mail: hang.wong@cityu.edu.hk).

Min Li is with the Department of Electronic and Computer Engineering, The Hong Kong University of Science and Technology, Hong Kong.

Bo Wang is with The State Key Laboratory of Terahertz and Millimeter Waves, Department of Electrical Engineering, City University of Hong Kong, Hong Kong. smartphones, smartwatches, smart wristbands, smart pencils, and smart glasses, are all potential IoT gateways at present and in the future. Many of these smart devices are small in size. Thus, they are required to equip with small-size multi-band antennas, supporting the need of various frequency bands with multiple communication protocols.

Dipole antenna is the simplest type of antenna from the theoretical point of view. It is widely applied in small wireless devices, especially those without a large-size ground. In order to cover more frequency bands, a lot of research has been conducted on multi-band dipole antennas. One common method is by adding more arms [2]-[4] or slots [5]-[7] on a dipole antenna for additional resonances. However, this method complicates the antenna structure and increases the antenna width. When even more frequency bands are required, the bulky structure is even unacceptable for many bar- or strip-shaped devices.

Another method is by utilizing the inherent harmonic modes of a dipole antenna [8]-[15]. Dipole antennas designed by this method generally have a more concise and compact structure.

We know the most common dipole antenna is with an approximate length of a half wavelength $(0.5 \lambda)$ and the feeding port at exactly the middle point. In this scheme, the dipole antenna operates with odd modes, which are also the odd-order modes: the $1^{\text {st }}$ mode $(0.5 \lambda$ mode $), 3^{\text {rd }}$ mode $(1.5 \lambda$ mode $), 5^{\text {th }}$ mode ( $2.5 \lambda$ mode), etc. However, the even-order modes (also the even modes $): 2^{\text {nd }}$ mode $(1 \lambda$ mode $), 4^{\text {th }}$ mode $(2 \lambda$ mode $), 6^{\text {th }}$ mode ( $3 \lambda$ mode), etc. are not excited and utilized due to their very high input impedance [16], [17]. They are also called antiresonance in the circuit model of antenna, which describes the condition that the reactance vanishes, but the resistance approaches infinity [18]. In [9], the dipole antenna excited the fundamental mode $(0.5 \lambda$ mode $)$ and the $1.5 \lambda$ mode. The resonant frequency of the higher mode could be tuned by the embedded slots. In [10], $0.5 \lambda$ and $1.5 \lambda$ modes were excited. The resonant frequency of $1.5 \lambda$ mode was tuned by pairs of stubs at the current nulls of this mode. In [11], $0.5 \lambda$ and $1.5 \lambda$ modes were excited. The resonant frequency of $0.5 \lambda$ mode could be tuned by the embedded capacitors, while the $1.5 \lambda$ mode kept unchanged. Thus, the two modes are compressed for wider bandwidth. In [12], an electrically small loop resonator was loaded in the middle point, so that the resonant frequencies of $0.5 \lambda$ and $1.5 \lambda$ modes approached. Thus, an omnidirectional radiation pattern in H-plane could be achieved over a wider bandwidth. In [8], $1.5 \lambda$ and $2.5 \lambda$ modes were utilized. The resonant frequency of the higher mode shifted close to the lower one by loading stubs for wider bandwidth and a higher gain of the antenna.

A method to change this scheme is by offsetting the feeding location. Then the $0.5 \lambda$ mode together with the $1 \lambda$ mode could be 
excited [13]-[15]. In [13], a meandered offset-fed dipole antenna with $0.5 \lambda$ and $1 \lambda$ modes was exhibited, in comparing with a symmetric-fed meandered dipole with $0.5 \lambda$ and $1.5 \lambda$ modes. As the very small size made the antenna highly capacitive, the self-inductance from the meandered arms was increased to balance it. In [14], $0.5 \lambda$ and $1 \lambda$ modes were excited and merged. By a step-shaped feed gap, both two modes exhibit omni-directional radiation patterns. In [15], the proposed antenna achieved the same function as [14] by shifting the antenna's feeding point away from the dipole center by means of adding a strip to one arm and embedding a slot to the other. However, for this method, the feeding port is no longer in a symmetrical location for every mode. Thus, the excitation of the modes higher than the $2^{\text {nd }}$ will become even more difficult, which will be analyzed in detail in the next section. For an intuitive comparison, all the above-mentioned multi-mode dipole antennas are summarized in Table I.

TABLE I

MULTI-MODE DIPOLE ANTENNAS

\begin{tabular}{|c|c|c|c|c|c|c|c|c|c|c|}
\hline \multirow{3}{*}[8]{} & \multicolumn{6}{|c|}{ Modes } & \multirow{3}{*}{ 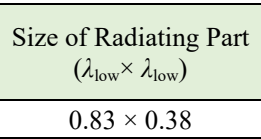 } & \multirow{2}{*}{\multicolumn{2}{|c|}{$\begin{array}{l}\text { Frequency bands } \\
\qquad(\mathrm{GHz})\end{array}$}} & \multirow[b]{2}{*}{ Efficiency } \\
\hline & \multirow{2}{*}{\multicolumn{2}{|c|}{$\begin{array}{cc}1^{\text {st }} & 2^{\text {nd }} \\
(0.5 \lambda) & (1 \lambda) \\
\end{array}$}} & \multirow{2}{*}{$\begin{array}{c}3^{\text {rd }} \\
(1.5 \lambda) \\
\checkmark\end{array}$} & \multirow[t]{2}{*}{$\begin{array}{l}4^{\text {th }} \\
(2 \lambda)\end{array}$} & \multirow{2}{*}{$\begin{array}{c}5^{\text {th }} \\
(2.5 \lambda) \\
\sqrt{ } \\
\end{array}$} & \multirow[t]{2}{*}{$\begin{array}{c}6^{\text {th }} \\
(3 \lambda)\end{array}$} & & & & \\
\hline & & & & & & & & - $3.30-3.60$ & $(8.7 \%)$ & - \\
\hline [9] & $\checkmark$ & & $\checkmark$ & & & & $0.43 \times 0.04$ & $\begin{array}{l}\text { - } 0.84-0.96 \\
\text { - } 2.33-2.51\end{array}$ & $\begin{array}{r}(13.0 \%) \\
(7.0 \%)\end{array}$ & - \\
\hline$[10]^{*}$ & $\checkmark$ & & $\checkmark$ & & & & $0.45 \times 0.17$ & - $1.63-2.71$ & $(49.7 \%)$ & avg. $88 \%$ \\
\hline [11] & $\checkmark$ & & $\checkmark$ & & & & $1.01 \times 0.09$ & - $1.88-3.18$ & $(51.4 \%)$ & - \\
\hline [12] & $\checkmark$ & & $\checkmark$ & & & & $0.45 \times 0.17$ & - $2.86-3.97$ & $(32.5 \%)$ & $\geq 79.7 \%$ \\
\hline$[13]^{* *}$ & $\checkmark$ & & $\checkmark$ & & $\checkmark$ & & $0.14 \times 0.02$ & $\begin{array}{l}-1.4 \\
-3.8 \\
-6.8 \\
\end{array}$ & & - \\
\hline$[13]^{* * *}$ & $\checkmark$ & $\checkmark$ & & & & & $0.14 \times 0.02$ & $\begin{array}{l}-1.1 \\
\bullet 1.4 \\
\end{array}$ & & - \\
\hline [14] & $\checkmark$ & $\checkmark$ & & & & & $0.36 \times 0.05$ & • $0.47-0.81$ & $(53.1 \%)$ & $>60 \%$ \\
\hline [15] & $\checkmark$ & $\checkmark$ & & & & & $0.36 \times 0.02$ & • $0.47-0.96$ & $(68.5 \%)$ & $>68 \%$ \\
\hline Proposed & $\checkmark$ & $\checkmark$ & $\checkmark$ & $\checkmark$ & $\checkmark$ & $\checkmark$ & $0.43 \times 0.03$ & $\begin{array}{l}0.73-0.91 \\
-1.48-1.72 \\
-2.29-3.67 \\
-3.85-4.02 \\
-4.59-5.01 \\
\end{array}$ & $\begin{array}{r}(22.0 \%) \\
(15.0 \%) \\
(46.3 \%) \\
(4.3 \%) \\
(8.8 \%) \\
\end{array}$ & $>80.4 \%$ \\
\hline
\end{tabular}

Our preliminary research on the multi-mode dipole antenna has been presented in EuCAP 2020 [19]. It was a dual-fed dipole antenna. One port is embedded in the middle point for exciting the odd modes. The other one, also in the middle point, is attached to a coupler for exciting the even modes. Furthermore, in this paper, a novel feeding structure combines these two ports into one. Thus, both odd and even modes could be excited by a single port. We know that for the multi-mode antennas, the difficulty of tuning harmonic frequencies increases dramatically as the utilized modes grow substantially. Thus, in order to manipulate such multiple modes, a novel method of tuning is proposed by combining antithetical structures of indentations and outdentations at different locations. By using the above techniques, the proposed antenna could excite all its first six modes, while covering five desired discrete frequency bands, including ISM bands of $868 \mathrm{MHz}$, $2450 \mathrm{MHz}$ for IoT, GNSS $1575 \mathrm{MHz}$ band (covering GPS L1, GLONASS L1, Galileo E1, and Beidou B1), 5G N78 band of $3500 \mathrm{MHz}$ and $\mathrm{N} 79$ band of $4900 \mathrm{MHz}$. For GNSS antennas, it is desirable to have circularly polarized radiation. However, the achievement of circular polarization usually relies on 2D structure [20]-[24]. For small multi-band antennas in mobile and IoT devices, the sizes are severely restricted. Thus, linearly polarized GNSS antennas are usually adopted [25]-[27].
The paper is organized as follows. Section II analyzes the characteristic mode properties of odd and even modes of any narrow strip in Part A, and then presents the method of excitation in Part B. Section III presents the method of tuning harmonic frequencies. Section IV presents the simulated and measured results of the proposed exemplified antenna.

\section{II.EXCITATION OF Both OdD AND EVEN ModeS}

\section{A. Characteristic mode properties of any narrow PEC strip}

The theory of characteristic mode (TCM) could bring physical insight into the radiating phenomena of antennas [28][30]. The characteristic modes are obtained by solving an eigenvalue equation derived from the method of moments (MoM): $\mathrm{X}\left(\mathbf{J}_{\mathrm{n}}\right)=\lambda_{\mathrm{n}} \mathrm{R}\left(\mathbf{J}_{\mathrm{n}}\right)$, Where $X$ and $R$ are the imaginary and real parts of an impedance matrix $Z$. $\mathbf{J}_{\mathrm{n}}$ is the eigencurrent of the $n$-th mode. $\lambda_{n}$ is the eigenvalue. The corresponding characteristic angle of this mode, $\theta_{\mathrm{n}}=180^{\circ}-\arctan \lambda_{\mathrm{n}}$. At a given frequency, we can determine whether or not the characteristic mode is at resonance by looking at its $\theta_{\mathrm{n}}$. When $\theta_{\mathrm{n}}$ is near $180^{\circ}$, the mode is at resonance. The real current $\mathbf{J}$ on a conductive surface $S$ can be expressed as a linear superposition of the normalized eigencurrents $\mathbf{J}=\sum \alpha_{\mathrm{n}} \mathbf{J}_{\mathrm{n}}$, where $\alpha_{\mathrm{n}}$ is the modal weighting coefficient (MWC) of the $n$-th characteristic 
mode, indicating how strongly $\mathbf{J}_{\mathrm{n}}$ is excited.

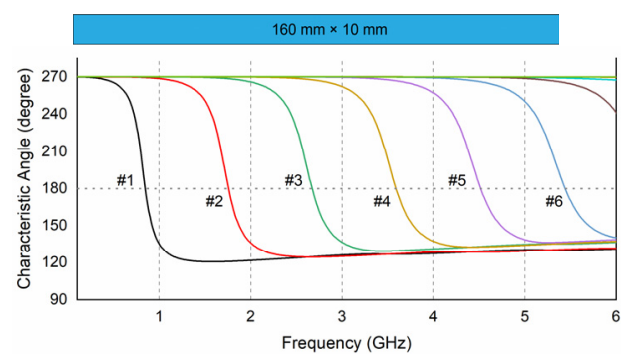

Fig. 1. Characteristic modes resonating below $6 \mathrm{GHz}$ for a narrow PEC strip

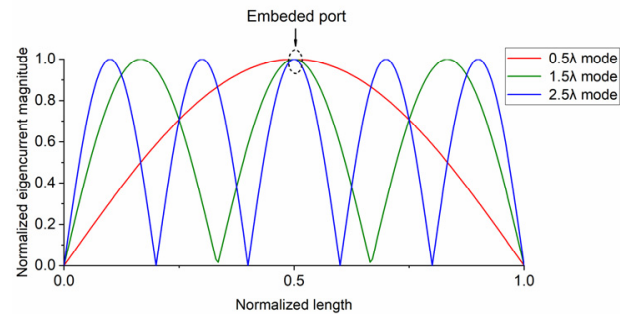

(a) Odd modes

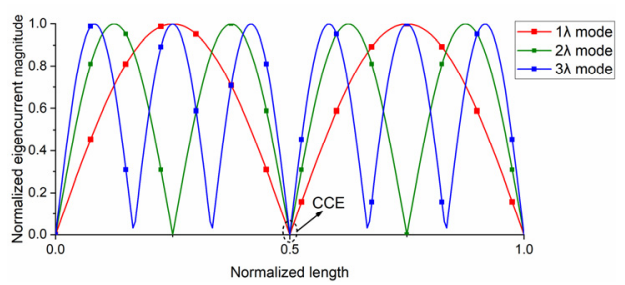

(b) Even modes

Fig. 2. Normalized eigencurrent magnitudes of the first six modes

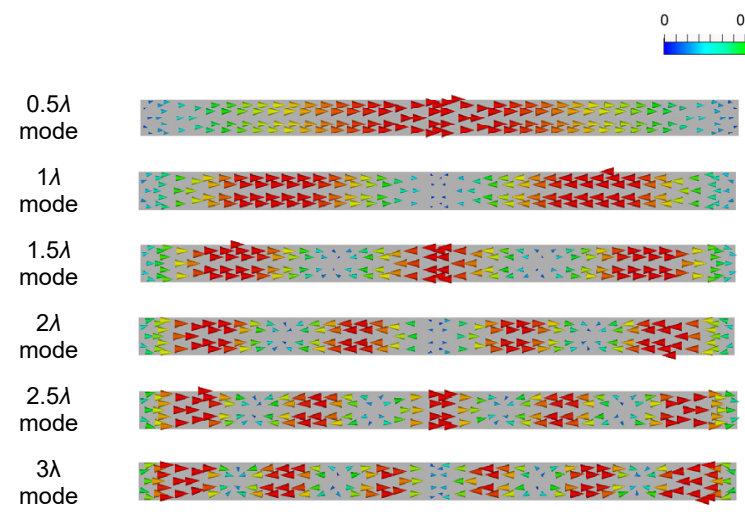

Fig. 3. Simulated eigencurrent of the first six modes of a narrow PEC strip

Then, we conduct characteristic mode analysis (CMA) on a narrow perfect electric conductor (PEC) strip by an in-house MATLAB program. Narrow means that all transverse modes of the strip could be neglected in the concerned frequency band. Here, we choose a size of $160 \mathrm{~mm} \times 10 \mathrm{~mm}$ for calculating. For other narrow PEC strips, the situation should be similar. Then the characteristic modes of the strip are exhibited with their characteristic angles in Fig. 1. The modes are numbered according to their resonant frequencies from lower to higher till $6 \mathrm{GHz}$. As can be seen, the characteristic modes are almost uniformly distributed in the spectrum. The $0.5 \lambda$ mode, resonating at around $868 \mathrm{MHz}$, is the fundamental and the most commonly used mode. The other modes are higher harmonic modes, from $2^{\text {nd }}$ to $6^{\text {th }}$ modes, resonating at approximate multiples of the fundamental frequency.

The eigencurrent of these six modes all exhibits sinusoidal distribution, as shown in Fig. 2. The y-axis shows the normalized eigencurrent magnitude (not amplitude), while the $\mathrm{x}$-axis shows the normalized length of the strip. The odd modes $(0.5 \lambda, 1.5 \lambda$, and $2.5 \lambda$ modes $)$ are all with eigencurrent maxima at the center of the strip, and eigencurrent nulls at the two ends of the strip, as in Fig. 2(a). The even modes $(1 \lambda, 2 \lambda$, and $3 \lambda$ modes) are all with eigencurrent nulls at the center and the two ends of the strip, as in Fig. 2(b). The results are also verified by the simulated eigencurrent distribution, shown in Fig. 3.

\section{B. Method of feeding}

For common dipole antennas, as shown in Fig. 4(a), the feeding port is embedded in the middle point of the strip, also the point of eigencurrent maximum. This scheme could excite the odd modes [31]. The odd modes' input impedance could be near $50 \Omega$. Thus they are matched. The even modes have a very high input impedance at this point, so could not be excited. The simulated reflection coefficient of this antenna is shown with the blue line in Fig. 8.

To excite the even modes, we could mount a capacitive coupling element (CCE) in the middle point [31], [19], as shown in Fig. 4(b). CCEs are usually much smaller than the radiator, non-resonant at concerned frequencies. They need to be placed at the voltage maxima of the eigencurrent of the radiator, which are also the eigencurrent minima, in the condition of a standing wave field. For this narrow strip, because the even modes have eigencurrent nulls in the middle point, they could be excited by a CCE. The reflection coefficient is shown with the green line in Fig. 8 .
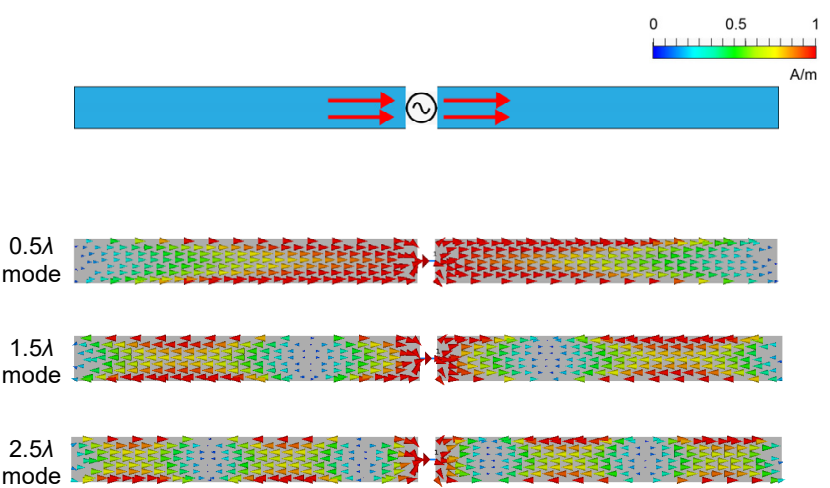

(a) Normalized current distribution of common dipole antenna with embedded port (odd modes)

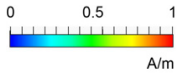

(2) 


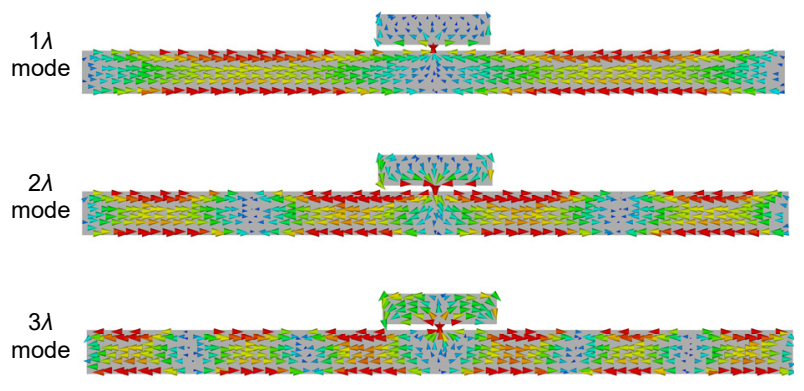

(b) Normalized current distribution of dipole antenna with CCE (even modes)

Fig. 4. Excitation of odd and even modes respectively (In the schematics, the red arrows denote the current, while small red circles denote the current nulls)

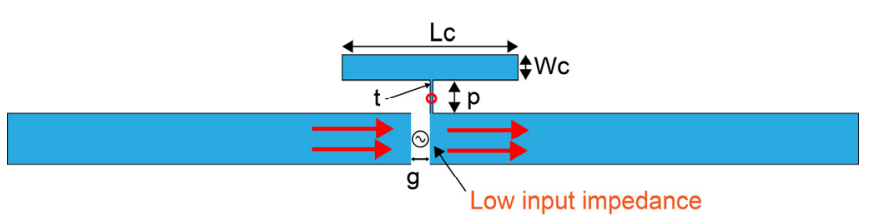

(a) Odd modes in the proposed structure

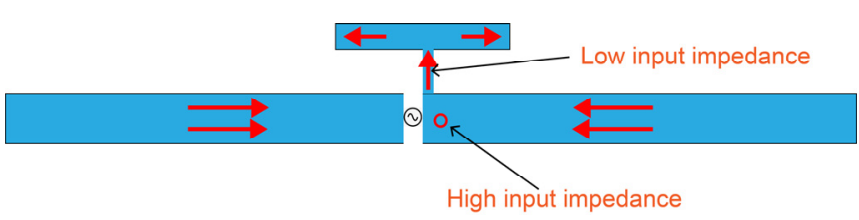

(b) Even modes in the proposed structure

Fig. 5. Excitation of odd and even modes simultaneously (Red arrows denote the real current, while small red circles denote the current nulls)

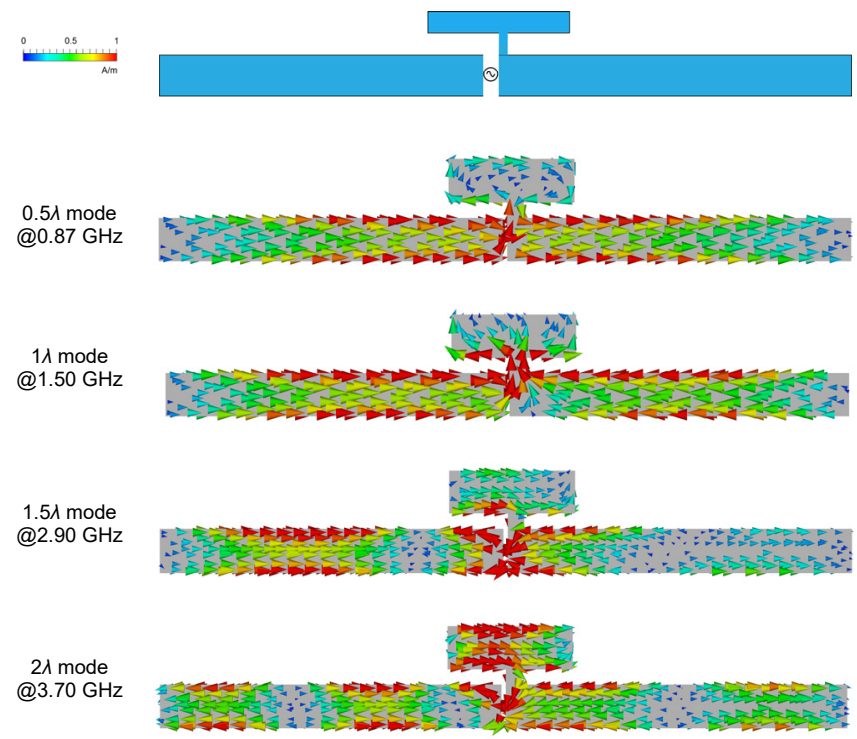

$2.5 \lambda$ mode @ $4.25 \mathrm{GHz}$ $3 \lambda$ mode
$@ 5.55 \mathrm{GHz}$
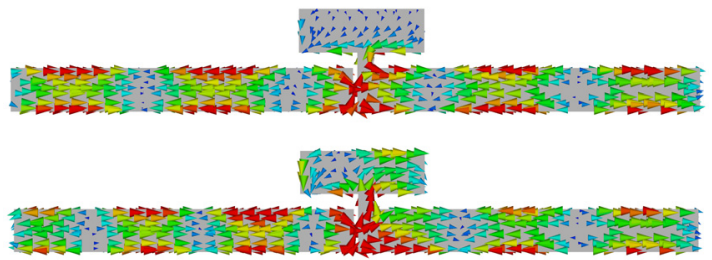

$3.5 \lambda$ mode @6.30 GHz

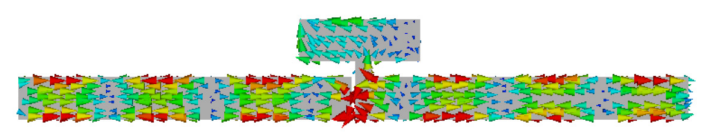

Fig. 6. Normalized current distribution of inherent resonant modes of a $160 \mathrm{~mm}$ $\times 10 \mathrm{~mm}$ dipole antenna

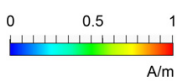

CCE mode @2.23 GHz

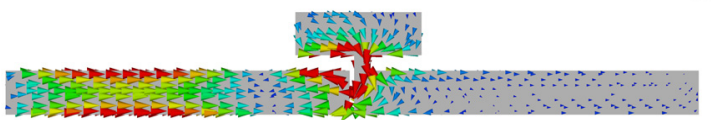

Fig. 7. Normalized current distribution of the CCE mode

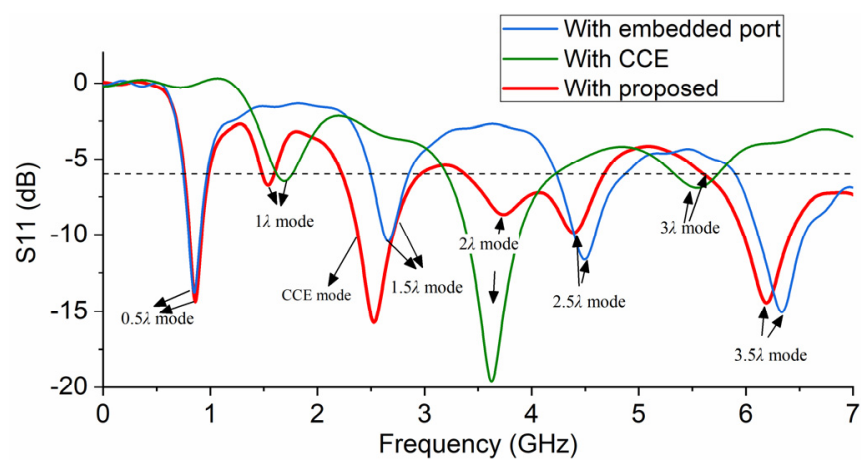

Fig. 8. Comparison of simulated reflection coefficients of antetype dipole antennas with three different feeding schemes (without balun)

TABLE II DIMENSIONS OF THE THREE ANTENNA ARCHETYPeS (Unit: $\mathrm{mm}$ )

\begin{tabular}{cccccccc}
\hline & $L$ & $W$ & $g$ & $t$ & $p$ & $L_{\mathrm{c}}$ & $W_{\mathrm{c}}$ \\
\hline Odd-mode dipole & 160 & 10 & 1 & - & - & - & - \\
\hline Even-mode dipole & 160 & 10 & - & - & 1 & 27.5 & 9 \\
\hline Odd- and even-mode dipole & 160 & 10 & 1 & 3.8 & 3.7 & 29.1 & 9.9 \\
\hline
\end{tabular}

In our research in [19], the proposed dipole antenna had two ports. One was embedded in the middle point of the dipole to excite the odd modes. The other port was attached to a CCE, also in the middle point, to excite the even modes. Here, we further combine these two ports into one. First, a T-shaped stub is mounted in the middle point, at one end of the embedded port, as in Fig. 5.

When this proposed structure operates in odd modes, only a very small current goes into the T-shaped stub, because the path of the strip has a low input impedance at the center. Then, the odd modes could still be excited. The T-shaped stub also has a very small impact on the resonant frequencies of the odd modes, which will be explained in detail in the next section. 
When the antenna operates in even modes, the path of the strip has a very high input impedance at the center. However, the path toward the T-shaped stub has a relatively low input impedance, because the T-shaped stub is very short compared to the strip, thus not in resonance in the concerned frequency band. So, the current mostly goes into the T-shaped stub. In this condition, the short strip on top of the T-shaped stub becomes an equivalent $\mathrm{CCE}$, which could excite the even modes of the strip.

The analysis could be verified by the simulated current distribution of the proposed structure after it is fed in the middle, as shown in Fig. 6. The dimensions of the above three antenna archetypes are all shown in Table II. They all adopt the same $160 \mathrm{~mm} \times 10 \mathrm{~mm}$ narrow PEC strip for an intuitive comparison. The reflection coefficient with this novel feeding structure is shown with the red line in Fig. 8. We can see that all the first several modes from $0.5 \lambda$ mode to $3 \lambda$ mode are all excited. However, apart from the desired inherent modes of the dipole antenna, there also exists an unplanned CCE mode resulted from the introduction of CCE. The simulated current distribution of this mode is shown in Fig. 7. The CCE and the left arm constitute a $1 \lambda$ dipole, thus the port could locate at about one-fourth to the right. It is an eigencurrent maximum, so the mode could be excited. The right arm of the dipole also has a smaller current because of the coupling effect. The CCE mode and the $1.5 \lambda$ mode are quasi-degenerate in this model. Their resonant frequencies are quite close. When the observing frequency is lower (2.23 GHz as in Fig. 7), the third resonance of the antenna behaves more as a CCE mode. When the observing frequency is higher $(2.90 \mathrm{GHz}$ as in Fig. 6), the resonance behaves more as a $1.5 \lambda$ mode.

\section{TUNING OF RESONANT FREQUENCIES}

We know that higher harmonic frequencies are in approximate multiples of the fundamental frequency. However, in practical applications of multi-band antennas, the required frequencies do not definitely conform to this relationship. So, tuning the resonant frequencies of the dipole antenna is necessary. In [19], the frequency tuning was achieved by two impedance matching circuits separately for odd and even modes. However, this method is indirect and increases the complexity of the circuits. In [11], the research found that the resonant frequency of a particular mode could be increased by loading capacitors. Previous research of [8], [10] found that inserting stubs at current nulls could lower down the resonant frequency of the corresponding mode. However, in the above research, the methods are only used to tune a single mode. It is not flexible enough to manipulate resonant frequencies of such multiple modes at the same time, as in the condition of ours.

In this paper, we consummate the existing research and present a more general method. It is by the combination of antithetical structures of inward dented shapes (indentations for short) and outward dented shapes (outdentations for short) at different locations. The principles are also intuitively explained by eigencurrent distributions.

Due to the skin effect, the current density in the fringe of the strip is much larger than that in the center. Thus, the fringe current dominates the current on the strip and decides the current path. So, the indentations and outdentations could have a completely opposite effect on tuning the modes. This could be observed intuitively by the eigencurrent distribution. The following results in Fig. 9 are from the structure simulation by the CMA solver without any sources.

In Fig. 9, the four PEC dipoles have the same dimension of $160 \mathrm{~mm} \times 10 \mathrm{~mm}$. The dipoles in (a) and (c) operate in $0.5 \lambda$ mode, while the dipoles in (b) and (d) operate in $1 \lambda$ mode. There are four typical conditions:

1) In Fig. 9(a), when a pair of indentations is at the eigencurrent maximum, the current path is elongated along the perimeter of the indentation. So, the resonant frequency is decreased.

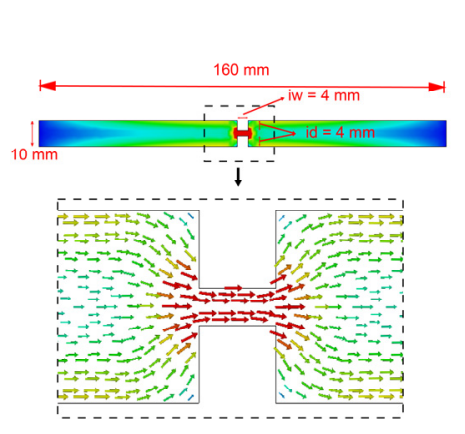

(a)

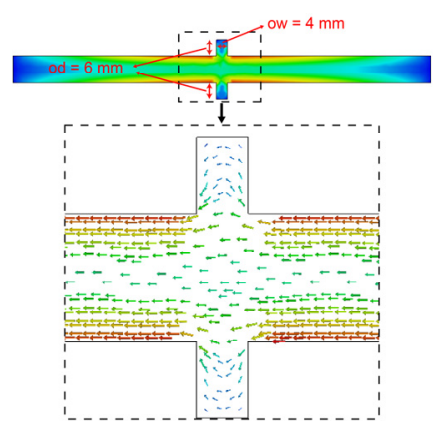

(c)

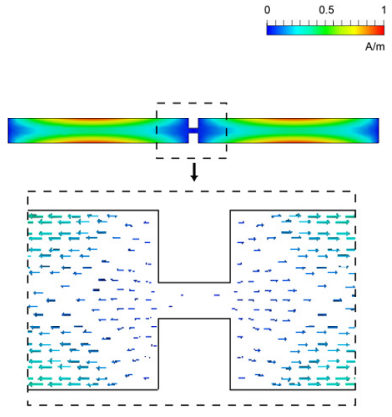

(b)

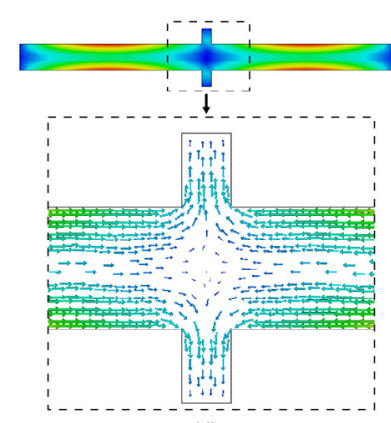

Fig. 9. Simulated eigencurrent distribution after inserting indentations at (a) eigencurrent maximum and (b) eigencurrent null; outdentations at (c) eigencurrent maximum and (d) eigencurrent null

2) When the indentation is at the eigencurrent minimum, the current path is not affected, because the magnitude of the current is approaching zero at this point, as shown in Fig. 9(b). So, the resonant frequency is invariant.

3) When the outdentation is at an eigencurrent maximum, the transverse currents inside the outdentation, coming from left and right, are in opposite directions, so counteracted. Thus, the current path is not affected, as shown in Fig. 9(c). So, the resonant frequency is invariant. That is also the reason that the additionally mounted T-shaped stub at the middle point of the proposed structure does not affect the resonant frequencies of the odd modes, which is introduced in the previous section.

4) When the outdentation is at an eigencurrent minimum, the directions of the currents from left and right are the same in the outdentation. So, the current nulls move to the vertices of the 
pair of outdentations. Thus, the current path is elongated, as shown in Fig. 9(d). So, the resonant frequency is decreased.

The above conclusions in the four conditions are also quantitatively verified by parameter sweeps in the form of characteristic angles. Fig. 10 exhibits the resonant frequency deviations when a pair of indentations is at the middle point of the strip, with the same dimensions in Fig. 9. When the length of the indentations id increases from 0 to $4 \mathrm{~mm}$, the resonant frequencies of the $0.5 \lambda, 1.5 \lambda$, and $2.5 \lambda$ modes decrease, because the indentation is at the eigencurrent maximum, as the condition in Fig. 9(a); the resonant frequencies of the $1 \lambda, 2 \lambda$, and $3 \lambda$ modes are invariant, because the indentation is at the current null for them, as the condition in Fig. 9(b).

When the length of the outdentation od increases from 0 to 8 $\mathrm{mm}$ in Fig. 11, the resonant frequencies of $0.5 \lambda, 1.5 \lambda$, and $2.5 \lambda$ modes are invariant, because the outdentation is at the current maximum, as the condition in Fig. 9(c); the resonant frequencies of the $1 \lambda, 2 \lambda$, and $3 \lambda$ modes decrease, because the outdentation is at the current null for them, as the condition in Fig. 9(d).

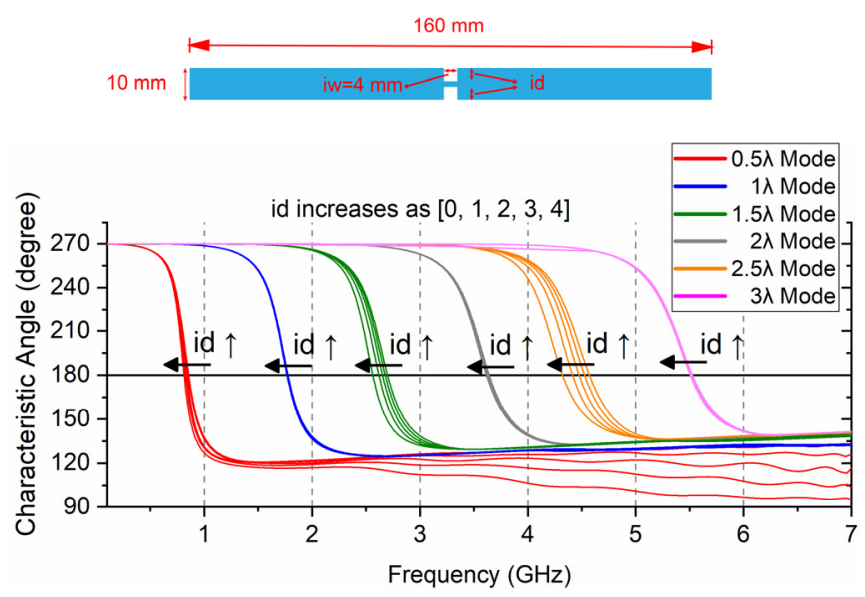

Fig. 10. Resonant frequency deviation when a pair of indentations is at the middle point of the strip (id from 0 to $4 \mathrm{~mm}$ )
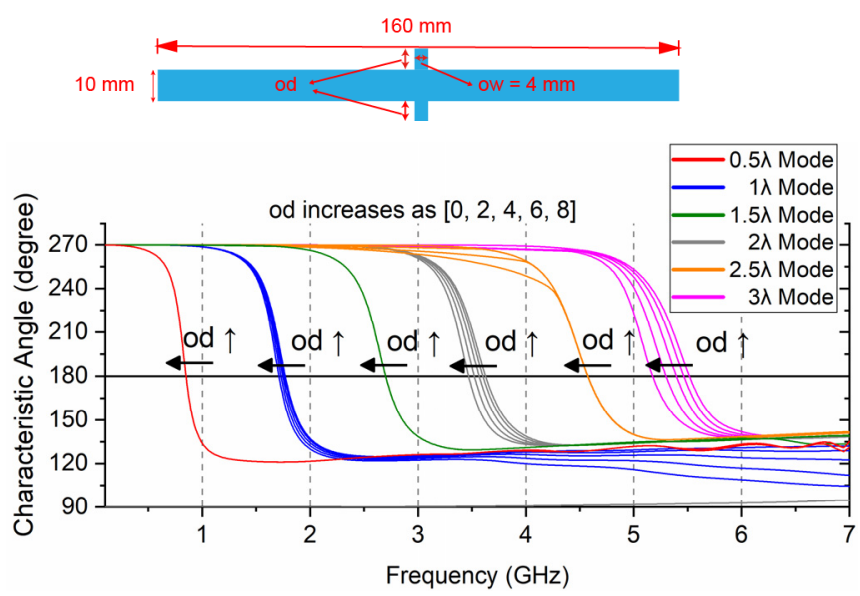

Fig. 11. Resonant frequency deviation when a pair of outdentations is at the middle point of the strip ( $o d$ from 0 to $8 \mathrm{~mm}$ )

It is noteworthy that, here the step of variation for the outdentations is twice as long as the indentations, then the former could have a similar tuning effect to the latter. It shows that the indentation's tuning effect is better than outdentations.

Moreover, due to the limitation of the width of the strip $W$, when the required length of indentation $i d$ exceeds half of the width $W / 2$, the indentations could rotate $90^{\circ}$ and continue extending along the axis direction.

We could also observe that when the resonant frequency is higher, the frequency deviation (if the mode has) tends to be more significant. That is because the length variation of the current path takes a larger percentage of the wavelength for a higher-order mode. Once again, we consider the condition that the indentations and outdentations are at the center of the strip. Then, we assume that the extended length of the current path $\Delta L$ is the same within all the odd modes $(n=1,3,5 \ldots)$ or within all the even modes $(n=2,4,6 \ldots)$. Then, the frequency deviation $\Delta f_{\mathrm{n}}$ is:

$$
\begin{aligned}
\Delta f_{\mathrm{n}} & =f_{\mathrm{n}}^{\prime}-f_{\mathrm{n}}=\frac{n c}{2(L+\Delta L)}-\frac{n c}{2 L} \\
& =\frac{n c}{2} \cdot\left(\frac{1}{L+\Delta L}-\frac{1}{L}\right) \propto n
\end{aligned}
$$

Where $f_{\mathrm{n}}{ }^{\prime}$ and $\lambda_{\mathrm{n}}{ }^{\prime}$ are the tuned resonant frequency and wavelength of the $n$-th mode. $f_{\mathrm{n}}$ and $\lambda_{\mathrm{n}}$ are the untuned resonant frequency and wavelength of the $n$-th mode. $L$ is the length of the strip. It shows, in this condition, the frequency deviation is proportional to the mode number $n$.

Indentations and outdentations in the middle point of the strip are the most obvious cases, because the eigencurrent at this location is either maximal or zero. Thus, the effect of tuning each mode is either maximized or none. However, the location is not confined to it. Indentations and outdentations take effect in varying degrees according to the eigencurrent magnitude. Each mode has its unique feature of eigencurrent maxima and minima distribution. Thus, by combining a series of indentations and outdentations at specific locations, we could particularly sort out the desired modes and tune them to the desired frequencies. For this reason, this method could be applied well in the conditions of tuning multiple resonant modes simultaneously.

\section{Simulated And Measured Results}

The schematic of the proposed antenna is shown in Fig. 12, with the dimensions in Table III. The substrate of RO4003C $\left(\varepsilon_{\mathrm{r}}\right.$ $=3.38$ and $h=0.8 \mathrm{~mm}$ ) is adopted for this antenna. In our proposed antenna, the dipole has a size of $151.5 \mathrm{~mm} \times 9 \mathrm{~mm}$, with the right arm on the front side and the left arm on the back side. Here, the CCE is connected to the right arm. The length of it $\left(L_{\mathrm{c}}\right)$ is $28 \mathrm{~mm} . L_{\mathrm{c}}$ is 0.270 times of one wavelength of 1.575 $\mathrm{GHz}, 0.592$ times of one wavelength of $3.45 \mathrm{GHz}$, and 0.841 times of one wavelength of $4.9 \mathrm{GHz}$. It is noteworthy that, if the operating frequency continues increasing to be very high, the CCE's capability of exciting the even modes will decline, because the electric field generated by the CCE will begin to be dissimilar to the eigenfield of the even-higher-order modes in the region between the CCE and the strip [32]. 
The feedline is in the form of a parallel stripline. The front side and back side have the same width $W_{\mathrm{f}}$. A balun is designed for the connection between the balanced antenna and the unbalanced typical $50 \Omega$ SMA connector. It is necessary for the accurate measurement of dipole antennas. Without the balun, the currents will flow back to the outer surface of the feeding cable, resulting in secondary radiation. The radiation patterns and the efficiency will be affected significantly [33]. For narrow-band dipole antennas, a compact quarter-wavelength sleeve balun could be adopted to provide an open circuit for the signal. For broad-band or multi-band dipole antennas, the feeding cable can be covered with an electro-magnetic interference (EMI) suppressant material to absorb unwanted EM radiation. By this method, the shapes of the simulated and measured radiation patterns will be similar. However, the antenna's radiation efficiency will decrease because of the energy absorbed by the EMI suppressant material [33].

Here, we adopt a large-size taper-shape balun to provide a multi-band balance-to-unbalance transformation. At the same time, the impedance matching could be further improved. However, it is noteworthy that this bulky balun is not necessary in practical small wireless devices. The RF transceiver chip could be directly mounted between the two arms.

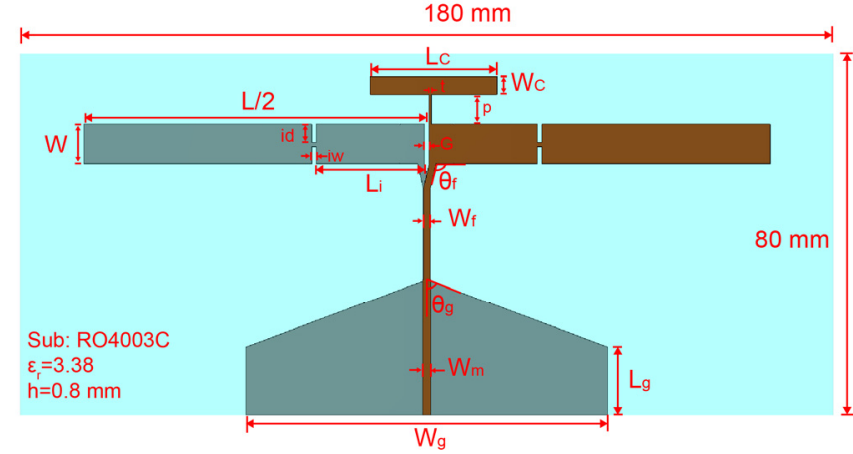

Fig. 12. Schematic of the proposed antenna ( front side, back side, substrate)

TABLE III

DIMENSIONS OF THE PROPOSED ANTENNA (Unit: $\mathrm{mm}$ )

\begin{tabular}{cccccccc}
\hline $\boldsymbol{G}$ & $\boldsymbol{i d}$ & $\boldsymbol{i w}$ & $\boldsymbol{L}$ & $\boldsymbol{L}_{\mathbf{C}}$ & $\boldsymbol{L}_{\boldsymbol{g}}$ & $\boldsymbol{L}_{\boldsymbol{i}}$ & $\boldsymbol{p}$ \\
\hline 0.5 & 4.3 & 2 & 151.5 & 28 & 15 & 24 & 6.5 \\
\hline $\boldsymbol{t}$ & $\boldsymbol{\theta}_{\boldsymbol{f}}$ & $\boldsymbol{\theta}_{\boldsymbol{g}}$ & $\boldsymbol{W}$ & $\boldsymbol{W}_{\mathbf{C}}$ & $\boldsymbol{W}_{\boldsymbol{f}}$ & $\boldsymbol{W}_{\boldsymbol{g}}$ & $\boldsymbol{W}_{\boldsymbol{m}}$ \\
\hline 0.5 & $103^{\circ}$ & $69^{\circ}$ & 9 & 4 & 1.5 & 80 & 1.8 \\
\hline
\end{tabular}

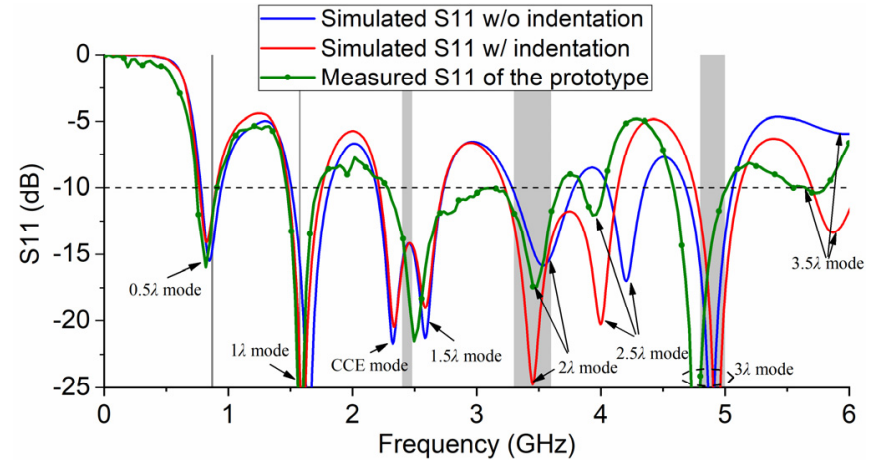

Fig. 13. Simulated and measured reflection coefficient of the proposed antenna (with balun)

The desired frequency bands of this antenna are ISM band 863 - $870 \mathrm{MHz}, 2400$ - $2480 \mathrm{MHz}$, GNSS 1559 - $1606 \mathrm{MHz}$ band (covering GPS L1, GLONASS L1, Galileo E1, and Beidou B1), 5G N78 band of $3300-3600 \mathrm{MHz}$, and 5G N79 band of $4800-5000 \mathrm{MHz}$. From the simulated result shown in Fig. 13, as expected, the dipole antenna has six frequency bands produced by the first six modes. However, we could see that the $2 \lambda$ mode is a little deviated from the $3300-3600 \mathrm{MHz}$ band, difficult to cover the whole band. Thus, we utilize the method of inserting indentations and outdentations to tune it while minimizing the impact on other modes.

For the $2 \lambda$ mode, we consider inserting two pairs of indentations $L / 6$ away from the middle point of the dipole antenna on the left and right arms. The locations are shown with the dashed lines in Fig. 14. Then, the resonant frequency of the $2 \lambda$ mode is decreased, because the eigencurrent magnitude nearly reaches one here. For the other modes, the $1.5 \lambda$ and $3 \lambda$ modes have their eigencurrent minima here, so they are not affected. The $2.5 \lambda$ mode is not utilized in this antenna. The $0.5 \lambda$ mode and $1 \lambda$ mode are tuned by the indentations because their eigencurrent magnitudes nearly reach one here. However, for these two lowest-order modes, the frequency deviations are much smaller than the $2 \lambda$ mode, as analyzed in the previous section. So, their frequency deviations are relatively small and acceptable. Then the simulated reflection coefficient with this pair of indentations is shown with the red line in Fig. 13. Thus, in this specific example, it happens that we could tune the antenna by only a pair of indentations, without inserting any more indentations or outdentations.

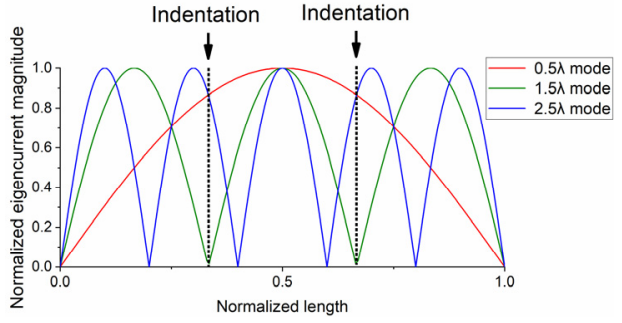

(a) Odd modes 


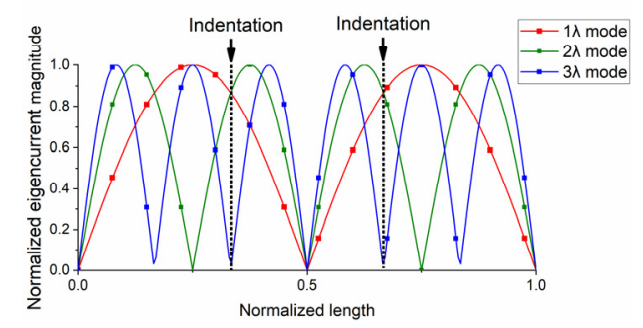

(b) Even modes

Fig. 14. Locations of indentations

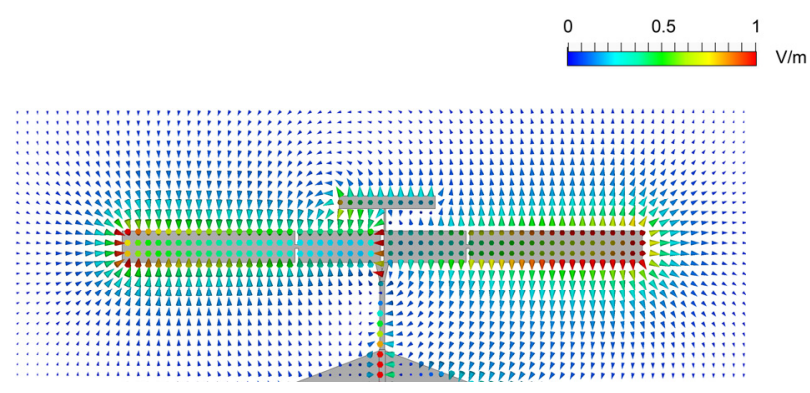

(a) $0.83 \mathrm{GHz}(0.5 \lambda$ mode)

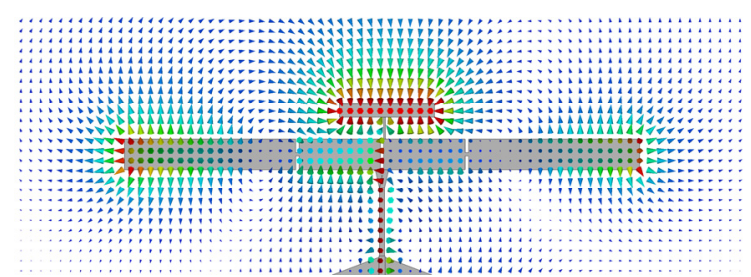

(b) $1.63 \mathrm{GHz}$ (1 $\lambda$ mode)

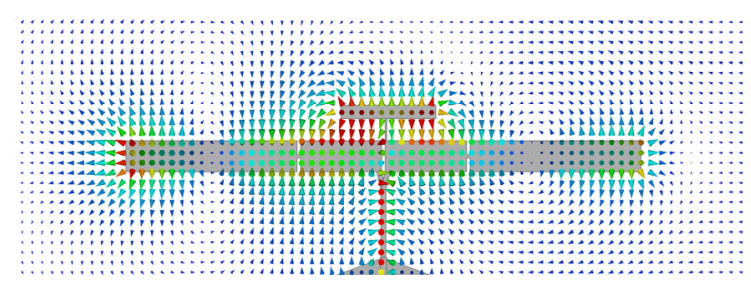

(c) $2.32 \mathrm{GHz}$ (CCE mode)

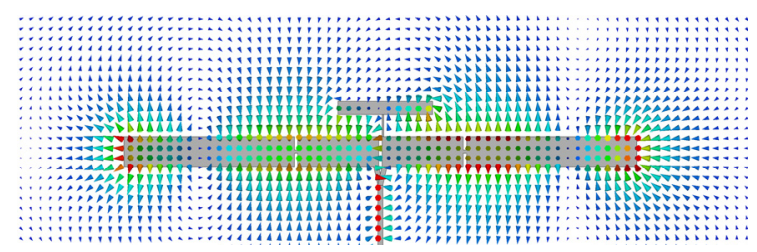

(d) $2.57 \mathrm{GHz} \quad$ (1.5^ mode)

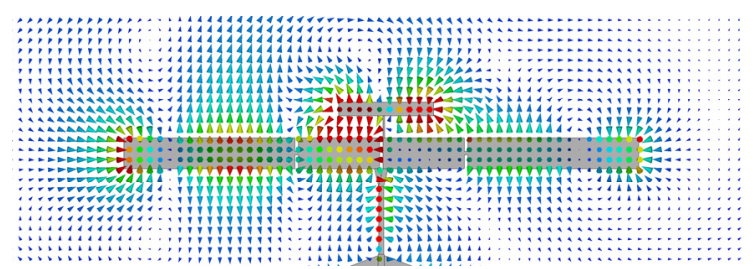

(d) $3.47 \mathrm{GHz} \quad(2 \lambda$ mode $)$

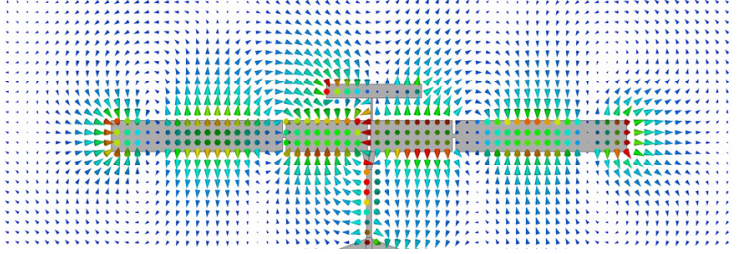

(e) $4.10 \mathrm{GHz}(2.5 \lambda$ mode)

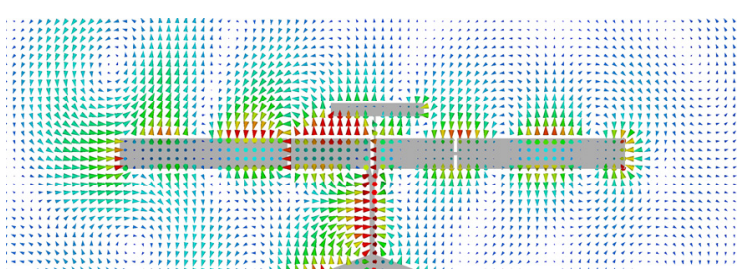

(f) $5.3 \mathrm{GHz}$ (3^ mode)

Fig. 15. Simulated Normalized E-field, $0.2 \mathrm{~mm}$ above the proposed dipole antenna
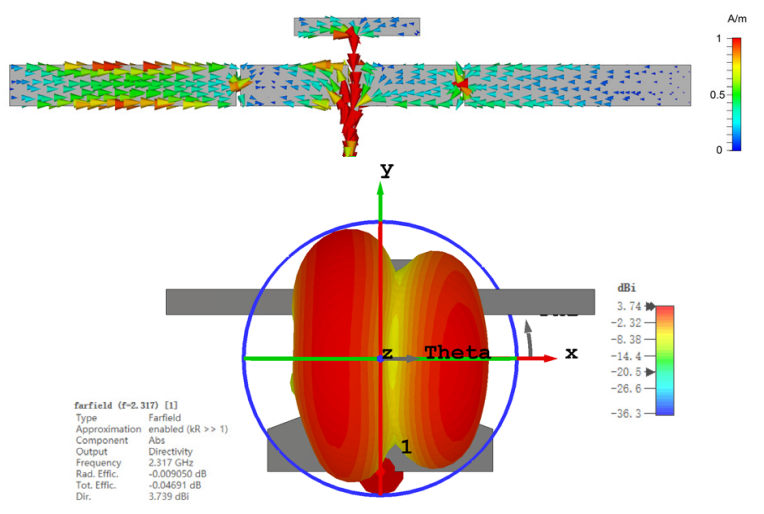

(a) $2.32 \mathrm{GHz}$ (CCE mode)
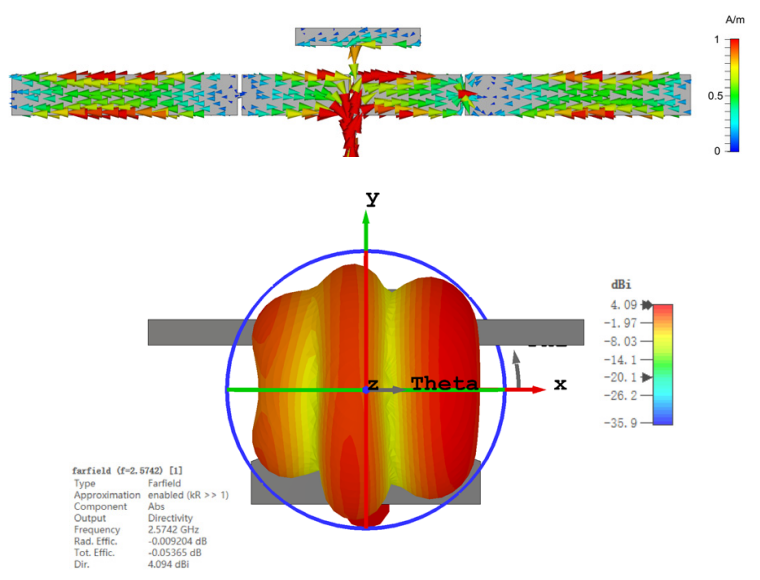

(b) $2.57 \mathrm{GHz}(1.5 \lambda$ mode)

Fig. 16. Simulated normalized current distribution and radiation patterns of CCE mode at $2.32 \mathrm{GHz}$ and $1.5 \lambda$ mode at $2.57 \mathrm{GHz}$

Afterward, we check the excitation of the first six modes by means of full-wave simulation. The result is shown with the red 
line in Fig. 13. Fig. 15 shows the simulated E-field distribution closely above the proposed antenna, exhibiting the multi-mode excitation. It is noteworthy that, in the simulated result of this structure, the CCE mode and the $1.5 \lambda$ mode turn to non-degenerate modes, due to the frequency deviation caused by introducing the balun. The current distribution and radiation patterns of these two modes are shown in Fig. 16. The CCE mode exhibits a $1 \lambda$-dipole-like radiation pattern because of the consistency of the current direction of the middle and right parts of the dipole.

Then the prototype, shown in Fig. 17, is measured by the vector network analyzer (VNA) and microwave anechoic chamber. As shown in Fig. 13, the measured reflection coefficient is lower than $-10 \mathrm{~dB}$ in the desired five frequency bands. The simulated and measured radiation patterns are shown in Fig. 18. It is noteworthy that for the even modes $(1 \lambda$, $2 \lambda$, and $3 \lambda$ modes), the CCE also has relatively strong radiation, which will obviously affect the radiation patterns, as shown in Fig. 15. Thus, the radiation nulls of the even modes at the YZ-plane could be improved.

Moreover, because we aim at exciting the intrinsic modes of a common narrow dipole antenna, the radiation patterns at each resonant frequency are quite close to the eigenfield of each resonant characteristic mode of a common narrow strip. Thus, the radiation patterns are not stable for different frequencies. For antennas of mobile devices, the stability of radiation patterns is usually not particularly stressed, because the orientation of the mobile devices themselves is not fixed [25] [27].

Then, the simulated and measured maximum gain and total efficiency are shown in Fig. 19. The measured efficiency is above $80.4 \%$ in the desired frequency bands.

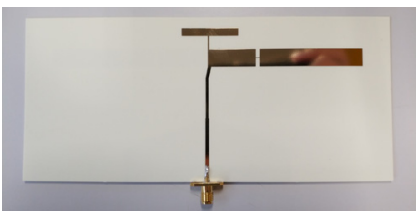

(a) Front side

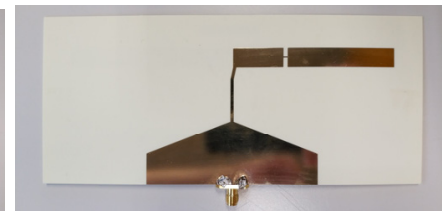

(b) Back side

Fig. 17. Prototype of the proposed antenna

- - Simulated Gain (Theta)
- - Simulated Gain (Phi) - Simulated Gain (Phi)
- Measured Gain (Theta)
Measured Gain (Phi)
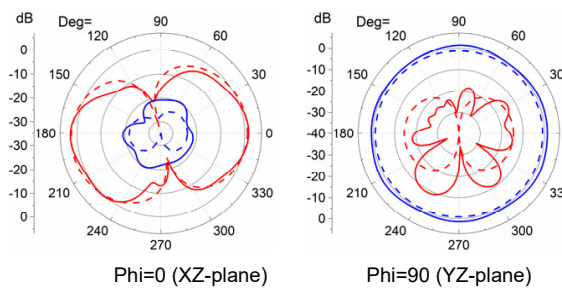

(a) $868 \mathrm{MHz}$
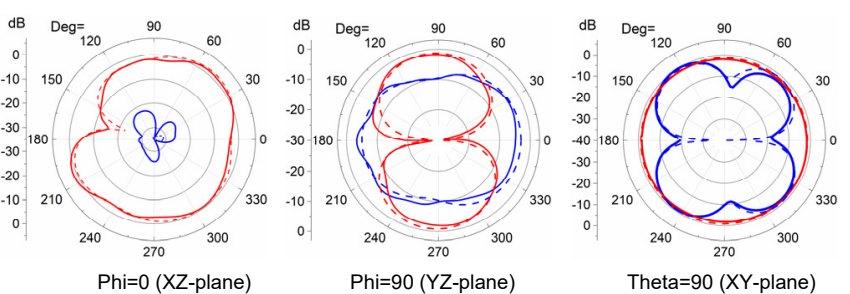

(b) $1575 \mathrm{MHz}$
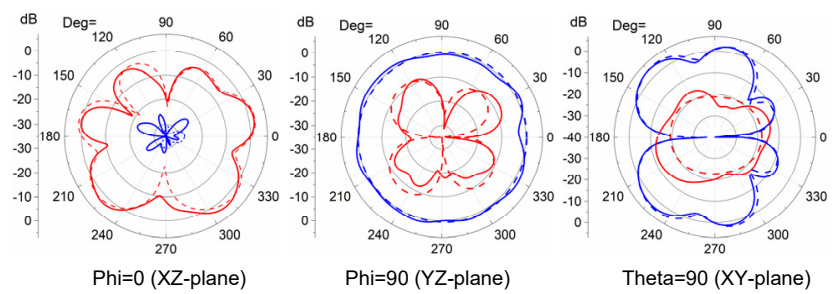

(c) $2450 \mathrm{MHz}$
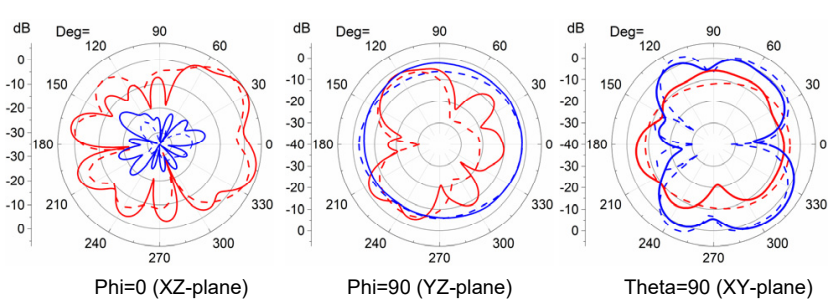

(d) $3450 \mathrm{MHz}$
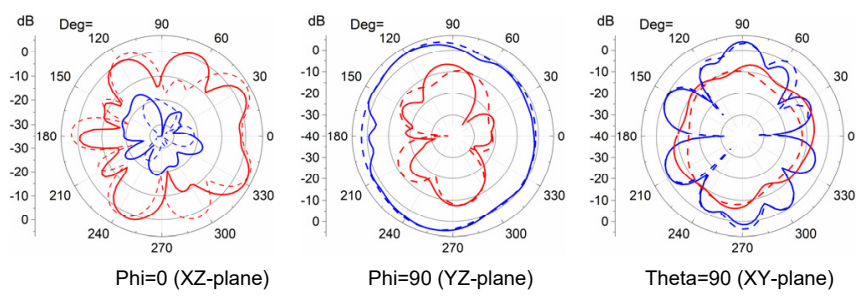

(e) $4000 \mathrm{MHz}$
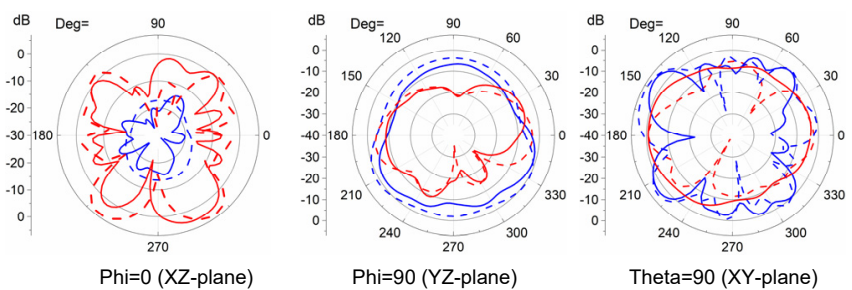

(f) $5300 \mathrm{MHz}$

Fig. 18. Simulated and measured radiation patterns at the six resonant frequencies

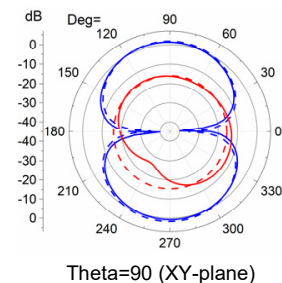

Theta $=90(X Y$-plane $)$ 


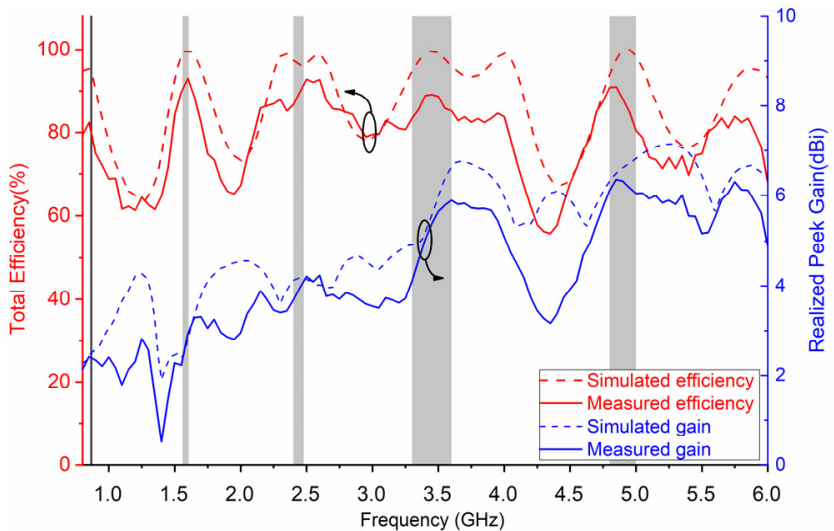

Fig. 19. Simulated and measured total efficiency and the maximum gain of the proposed antenna

\section{V.CONCLUSION}

In this paper, both odd and even modes of a dipole antenna are excited simultaneously by a single port by means of a novel feeding structure. Moreover, a novel method to tune the resonant frequencies of such multiple modes is proposed. Thus, all the first six modes of a narrow dipole antenna could be excited, among which five modes are utilized for IoT applications. This research could broaden the applications of the classic dipole antennas.

\section{References}

11] T. Zachariah, N. Klugman, B. Campbell, J. Adkins, N. Jackson, and P. Dutta, "The internet of things has a gateway problem," in Proceedings of the 16th international workshop on mobile computing systems and applications, 2015, pp. 27-32.

[2] H.-M. Chen, J.-M. Chen, P.-S. Cheng, and Y.-F. Lin, "Feed for dual-band printed dipole antenna," Electronics Letters, vol. 40, no. 21, pp. 1320 1322, 2004.

[3] Y.-H. Suh and K. Chang, "Low cost microstrip-fed dual frequency printed dipole antenna for wireless communications," Electronics Letters, vol. 36, no. 14, pp. 1177-1179, 2000.

[4] T.-L. Chen, "Multi-band printed sleeve dipole antenna," Electronics Letters, vol. 39, no. 1, pp. 14-15, 2003.

[5] Chih-Ming Su, Hong-Twu Chen, and Kin-Lu Wong, "Printed dual-band dipole antenna with U-slotted arms for 2.4/5.2 GHz WLAN operation," Electronics Letters, vol. 38, no. 22, pp. 1308-1309, Oct. 2002, doi: 10.1049/el:20020919.

[6] S.-Y. Chen, Y.-C. Chen, and P. Hsu, "CPW-fed aperture-coupled slot dipole antenna for tri-band operation," IEEE Antennas and Wireless Propagation Letters, vol. 7, pp. 535-537, 2008.

[7] V.-A. Nguyen, B.-Y. Park, S.-O. Park, and G. Yoon, "A planar dipole for multiband antenna systems with self-balanced impedance," IEEE Antennas and Wireless Propagation Letters, vol. 13, pp. 1632-1635, 2014.

[8] Y. Luo, Z. N. Chen, and K. Ma, "Enhanced bandwidth and directivity of a dual-mode compressed high-order mode stub-loaded dipole using characteristic mode analysis," IEEE Transactions on Antennas and Propagation, vol. 67, no. 3, pp. 1922-1925, 2019.

[9] S. Jeon, Y. Yu, and J. Choi, "Dual-band slot-coupled dipole antenna for $900 \mathrm{MHz}$ and $2.45 \mathrm{GHz}$ RFID tag application," Electronics letters, vol. 42, no. 22, pp. 1259-1260, 2006.

[10] W.-J. Lu, L. Zhu, K. W. Tam, and H.-B. Zhu, "Wideband dipole antenna using multi-mode resonance concept," International Journal of Microwave and Wireless Technologies, vol. 9, no. 2, pp. 365-371, 2017.
[11] H. Li and Y. Li, "Mode Compression Method for Wideband Dipole Antenna by Dual-point Capacitive Loadings," IEEE Transactions on Antennas and Propagation, 2020.

[12] W. Zhang, Y. Li, Z. Zhou, and Z. Zhang, "Dual-mode compression of dipole antenna by loading electrically small loop resonator," IEEE Transactions on Antennas and Propagation, vol. 68, no. 4, pp. 32433247,2019

[13] P. M. Izdebski, H. Rajagopalan, and Y. Rahmat-Samii, "Conformal Ingestible Capsule Antenna: A Novel Chandelier Meandered Design,” IEEE Transactions on Antennas and Propagation, vol. 57, no. 4, pp. 900-909, Apr. 2009, doi: 10.1109/TAP.2009.2014598.

[14] Y.-W. Chi, K.-L. Wong, and S.-W. Su, "Broadband printed dipole antenna with a step-shaped feed gap for DTV signal reception," IEEE Transactions on Antennas and Propagation, vol. 55, no. 11, pp. 33533356, 2007.

[15] C.-T. Lee and K.-L. Wong, "Broadband planar dipole antenna for DTV/GSM operation," Microwave and Optical Technology Letters, vol. 50, no. 7, pp. 1900-1905, 2008.

[16] W. L. Stutzman and G. A. Thiele, Antenna theory and design, 2nd Edition. P170. John Wiley \& Sons, 1996.

[17] Z. Zhang, Antenna design for mobile devices. P67. John Wiley \& Sons, 2017.

[18] A. D. Yaghjian and S. R. Best, "Impedance, bandwidth, and Q of antennas," IEEE Transactions on Antennas and Propagation, vol. 53, no. 4, pp. 1298-1324, 2005, doi: 10.1109/TAP.2005.844443.

[19] B. Xiao, H. Wong, and K. L. Yeung, "Penta-band Dual-fed Smart Glasses IoT Antenna," in 2020 14th European Conference on Antennas and Propagation (EuCAP), Mar. 2020, pp. 1-4. doi: 10.23919/EuCAP48036.2020.9136030.

[20] B. Y. Toh, R. Cahill, and V. F. Fusco, "Understanding and measuring circular polarization," IEEE Transactions on Education, vol. 46, no. 3, pp. 313-318, Aug. 2003, doi: 10.1109/TE.2003.813519.

[21] H. Wong, K.-M. Luk, C. H. Chan, Q. Xue, K. K. So, and H. W. Lai, "Small antennas in wireless communications," Proceedings of the IEEE, vol. 100, no. 7, pp. 2109-2121, 2012.

[22] C. F. Zhou and S. W. Cheung, "A Wideband CP Crossed Slot Antenna Using 1- $\lambda$ Resonant Mode With Single Feeding," IEEE Transactions on Antennas and Propagation, vol. 65, no. 8, pp. 4268-4273, Aug. 2017, doi: 10.1109/TAP.2017.2708101.

[23] Y. Li, Y. Wang, and K. Yu, "A single-band and dual-band circular polarized antenna by using asymmetric-circular shaped slots," in 2016 IEEE 5th Asia-Pacific Conference on Antennas and Propagation (APCAP), Jul. 2016, pp. 65-66. doi: 10.1109/APCAP.2016.7843101.

[24] D. Feng, H. Zhai, L. Xi, S. Yang, K. Zhang, and D. Yang, "A Broadband Low-Profile Circular-Polarized Antenna on an AMC Reflector," IEEE Antennas and Wireless Propagation Letters, vol. 16, pp. 2840-2843, 2017, doi: 10.1109/LAWP.2017.2749246.

[25] B. Xiao, H. Wong, D. Wu, and K. L. Yeung, "Metal-Frame Antenna for Big-Screen Smartphones Using Characteristic Mode Analysis," IEEE Access, vol. 7, pp. 122224-122231, 2019, doi: 10.1109/ACCESS.2019.2935474.

[26] Y. Li, Z. Zhang, J. Zheng, Z. Feng, and M. F. Iskander, "A Compact Hepta-Band Loop-Inverted F Reconfigurable Antenna for Mobile Phone," IEEE Transactions on Antennas and Propagation, vol. 60, no. 1, pp. 389-392, Jan. 2012, doi: 10.1109/TAP.2011.2167949.

[27] M. Jeon, W. C. Choi, and Y. J. Yoon, "GPS, Bluetooth and Wi-Fi tri-band antenna on metal frame of smartwatch," in 2016 IEEE International Symposium on Antennas and Propagation (APSURSI), 2016, pp. 2177-2178.

[28] R. J. Garbacz, "Modal expansions for resonance scattering phenomena," Proceedings of the IEEE, vol. 53, no. 8, pp. 856-864, 1965.

[29] R. Harrington and J. Mautz, "Theory of characteristic modes for conducting bodies," IEEE Transactions on Antennas and Propagation, vol. 19 , no. 5 , pp. $622-628,1971$.

[30] M. Cabedo-Fabres, E. Antonino-Daviu, A. Valero-Nogueira, and M. F. Bataller, "The theory of characteristic modes revisited: A contribution to the design of antennas for modern applications," IEEE Antennas and Propagation Magazine, vol. 49, no. 5, pp. 52-68, 2007.

[31] R. Martens, E. Safin, and D. Manteuffel, "Inductive and capacitive excitation of the characteristic modes of small terminals," in Antennas and Propagation Conference (LAPC), 2011 Loughborough, 2011, pp. 14.

[32] H. Aliakbari Abar and B. K. Lau, "Impact of capacitive coupling element design on antenna bandwidth," in European Conference on Antennas and Propagation (EuCAP), 2018, 2018, no. CP741. 
[33] L. Liu, S. W. Cheung, Y. F. Weng, T. I. Yuk, and M. Matin, "Cable effects on measuring small planar UWB monopole antennas," in Ultra Wideband -Current Status and Future Trends, InTech, 2012. 